\title{
A few words from Dr. Y. Shirley Meng, the new Editor-in-Chief of MRS Energy \& Sustainability
}

As most of the world continues to transition from an industrial to an information-driven society, the technological, sociological, and environmental requirements placed upon our energy infrastructure have become increasingly demanding. Renewable energy, distributed energy, portable energy, and smart energy systems are reshaping a future that will be more complex and potentially more volatile than the fossil fuel-based systems currently in place. Nonetheless, this future may bring with it far lower energy prices.

Materials have always played a critical role in energy production, conversion and storage, and are facing greater challenges today to meet higher performance and cost demands. MRS Energy \& Sustainability (MRS E\&S) launched in 2014 at the perfect time. This journal was designed to publish reviews on key topics in materials research and development as they relate to energy and sustainability. I am honored to be the new Editor-in-Chief starting in June 2019, as MRS E\&S ramps up its efforts to position itself as a unique journal that not only reports breakthroughs in materials science and energy technology, but also discusses the socio-economic and environmental impacts of these advances.

MRS E\&S's intended readership is a broad spectrum of scientists, academics, policy makers and industry professionals, all interested in the interdisciplinary nature of the science, technology and policy aspects of energy and sustainability. Few journals have the strong base and platform (readership and annual conferences) that supports MRS E\&S: The Materials Research Society (MRS) is a member-driven organization of approximately 14,000 materials researchers from academia, industry and government, and a breadth of energy and sustainability topics, particularly those with a materials focus, are featured at each MRS Spring and Fall meeting.

I am a materials scientist and engineer by training (CV) and have devoted my research efforts toward developing new materials and manipulating their interfaces and architectures for better electrochemical energy storage technologies, such as lithium ion batteries (LIB). The sustainability issue in LIB is always newsworthy as the LIB market worldwide has reached US\$30B in 2018 and is predicted to exceed US\$90B in $2025 .{ }^{1}$ This tremendous growth will stress the demand of materials containing lithium, cobalt and nickel. Recycling these materials will become imperative, and efforts in this regard are already in progress.

In 2015, I founded the Sustainable Power and Energy Center (SPEC) at UC San Diego, bringing together leading expertise from interdisciplinary fields focusing on breakthroughs in enabling distributed energy generation and storage, their integration-management systems, and the accompanying economics and social policies. MRS E\&S's original mission coincides perfectly with my own aspiration-to provide an integrated context of scientific, technological and sociological complexities relating to energy, the environment and sustainability. Over the past five years, MRS E\&S has published Reviews, Commentaries, Perspectives and Analysis from across the full range of disciplines concerned with energy and sustainability. My own list of must-read articles can be found here (Grid storage and LIB Recycling). Moving forward, we also welcome proposals for Original Research Articles that emphasize aspects of materials research and their sustainability and life-cycle assessment.

Any successful journal is characterized by a dedicated team of staff, a fair and rigorous peer-review process, high standards of copy-editing and production, swift publication, and editorial independence. These features, together with the journal's integration with MRS and the broader materials community support my goal for MRS E\&S to become a top journal for energy materials research that showcases the convergence between science, technology, economics and policy.

\section{REFERENCE:}

1. Lithium-ion battery market analysis by product (lithium cobalt oxide, lithium iron phosphate, NCA, LMO, LTO, lithium nickel manganese cobalt (NMC)), by application, and segment forecasts, 2018-2025 (2017). Available at: https://www.grandviewresearch.com/ industry-analysis/lithium-ion-battery-market (accessed June 28, 2019). 